\title{
Performance comparison of hydraulic and gravitation HybridICE filters in freeze desalination of mine waters
}

\author{
A Adeniyi ${ }^{*}$, RKK Mbaya', API Popoola', JP Maree ${ }^{2}$ and CM Zvinowanda ${ }^{2}$ \\ 'Department of Chemical, Metallurgical and Materials Engineering, Tshwane University of Technology, PMB X680 Pretoria 0001 \\ ${ }^{2}$ Department of Environmental, Water and Earth Sciences, Tshwane University of Technology, PMB X680 Pretoria 0001
}

\begin{abstract}
HybridICE is an emerging freeze desalination technology for treating complex mine wastewaters. The technology works on the principle that growing ice crystals reject impurities during freezing. The bottleneck in the freeze desalination processes may be the separation of ice from the ice slurry generated in the freeze engine. Two types of HybridICE filter have been developed to effect ice separation from ice slurry. The two types differ in the design of the filter elements, mode of feeding the slurry into the filter, and the mechanism of separation of ice from the slurry. In both types of filter, an extruded continuous ice column is formed around the filtering element, which has some openings to allow excess concentrated process water to flow out of the filter. However, the driving force in the gravitation filter is buoyancy, while in the hydraulic filter the ice column is driven by the pressure generated from the flow of the slurry. Salt removal and ice yield from each of the filter types was evaluated when a solution of approximately $4 \% \mathrm{~m} / \mathrm{m} \mathrm{NaCl}$ solution, prepared by dissolving $25.1 \mathrm{~kg}$ of $\mathrm{NaCl}$ in 674 litres of water, was treated in a HybridICE freeze crystallisation pilot plant. The objective was to describe the operation of the two types of filter and compare their performance. Salt removal and ice yield were found to be higher with the gravitation filter than the hydraulic filter.
\end{abstract}

Keywords: freeze, desalination, filter, yield, salt removal, ice

\section{INTRODUCTION}

The freezing process expels dissolved salt from water crystals, so that melting the ice crystals will produce fresh water. This provides one way in which wastewater can be treated to produce pure water, a technique known as freeze desalination (Clayton, 2011). There is a need to find alternative processes of desalination, and freezing is being considered as one of the more interesting methods (Cerci et al., 2003). One major reason is that the energy requirement for freezing processes is far lower than for evaporative processes. The latent heat of fusion of ice is only $334 \mathrm{~kJ} / \mathrm{kg}$ compared to the latent heat evaporation of water being $2340 \mathrm{~kJ} / \mathrm{kg}$ (Qin et al., 2008). One important difference between freezing processes and evaporation processes is that the former require mechanical power while the latter require heat. Advantages of freezing over other methods of desalination include minimisation of inevitable thermodynamic losses in heat exchange. Wastewater containing toxic compounds, heavy metals and organic compounds which are difficult to treat by conventional methods can be treated by freeze desalination (Mtombeni et al., 2013). The ice crystals produced are excellent latent heat storage material capable of storing cold energy of high density. Freeze desalination enables concentration of approximately $80 \%$ of the dissolved compounds in $25 \%$ of the original process water volume (Maurer et al., 2006). Due to low operating temperatures, low cost materials can be used in building freeze desalination plants, minimising capital costs and corrosion potential (Mtombeni et al., 2013).

This paper was originally presented at the 2014 Water Institute of Southern Africa (WISA) Biennial Conference, Mbombela, 25-29 May 2014.

* To whom all correspondence should be addressed.

ฮ +27 78924 0238; e-mail: adeniyia@tut.ac.za

\section{Challenges in freeze desalination processes}

Before the ice crystals are melted they must be separated from the slurry. Although ice crystals themselves are pure, salts adhere to the surface of the crystals, and one of the difficulties experienced is the economic removal, by washing, of the surface salts (Rahman et al., 2006). By having suitable shapes and sizes of crystals, the ratio of the surface area to the volume of ice crystal is minimised, thus facilitating the washing process (Shone, 1987). Brine tends to attach itself to the surface of ice crystals due to surface tension, which is higher with smaller crystal sizes, and this is why larger and more uniformly-sized ice crystals are preferred to small, fine crystals for effective separation of ice from slurry ( $\mathrm{Lu}$ and $\mathrm{Xu}, 2010)$. The salinity and temperature of the ice uniquely determine the amount of liquid brine and solid salts present (Cox and Weeks, 1975). Both the concentration and the microstructural location of impurities can have significant impacts on recrystallisation, grain growth, electrical conductivity and diffusion in ice (Baker et al., 2003). Few compounds can be incorporated directly into the ice crystals; hence, any impurity entrapment would be due to trapped solutions or trapped salt crystals and not direct incorporation into the crystal lattice (Olsson, 1999; Lorain et al., 2000).

The purpose of this work was to describe the operation of gravitation and hydraulic HybridICE filters, and compare their performance, in terms of salt removal and ice yield, in the separation of ice from slurry during freeze desalination of mine waters.

\section{MATERIALS AND METHODS}

\section{Feedstock}

The salt solution used for the experiments was approximately $4 \%$ mass by mass $\mathrm{NaCl}$ solution prepared by dissolving $25.1 \mathrm{~kg}$ $\mathrm{NaCl}$ (Sigma Aldrich) in $674 \mathrm{\ell}$ tap water. The mean electrical conductivity of the solution was $52.3 \mathrm{mS} / \mathrm{cm}$. 


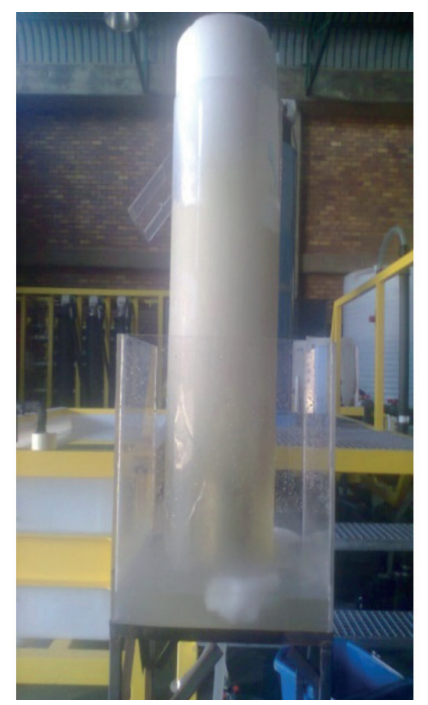

Figure 1

Gravitation HybridICE filter in operation

\section{Apparatus}

All freeze desalination methods involve crystallisation, separation, surface washing and melting of ice. The exception is the HybridICE technology which does not include surface washing of the ice crystals. HybridICE technology includes a refrigeration unit where a primary refrigerant (R404a) is recycled between the refrigeration unit and the scraped surface heat exchangers (SSHEs). The slurry of ice, which is a heterogeneous mixture of solid ice and cold, concentrated process water, is formed in the heat exchangers and delivered to the filter by a centrifugal pump. Two types of HybridICE filters have been developed, namely, gravitation and hydraulic. The two types differ in the following aspects:

- The construction of the filter element

- The mode of feeding the slurry into the filter

- The mechanism for separating the ice from the slurry in the filter.

\section{Gravitation HybridICE filter}

The gravitation HybridICE filter used for the experiment was constructed for a flow rate of $10 \mathrm{l} / \mathrm{min}$ based on the design equations developed by Adeniyi et al. (2013a).

$$
\begin{aligned}
& D=\sqrt[3]{3.64 Q} \\
& h_{i}=1.63 D \\
& H=1.92 h_{i} \\
& h_{s}=0.92 h_{i}
\end{aligned}
$$

Where:

$D$ is the diameter of the filter, $Q$ is the flow rate of the process wastewater, $H$ is the height of the filter, $h_{\mathrm{i}}$ is the height of the ice bed, $h_{\mathrm{s}}$ is the height of the slurry section which also indicates the position where the perforation begins on the filtering element.

Figure 2 shows a sketch of the filter element in the gravitation filter. There is a perforation section on the element which is positioned in such a way as to create the slurry section, where ice is separated by buoyancy force, and the concentrated process water flows out. Figure 3 shows how slurry is fed into the filter. There is 1 inlet pipe for the slurry and 1 outlet pipe for the concentrated process water. This is adequate for the slurry to flow in until it reaches the perforation, where the ice floats and is retained while the concentrated process water flows out.

\section{Separation mechanism}

When the ice slurry is pumped into the filter, there is continuous formation of an ice column around the filtering element starting from the perforation on the filtering element. The ice component of the slurry floats at the perforation on the filtering element due to buoyancy forces while the concentrated process water flows out through the filtering element. This is because ice is less dense than water. The ice forms into an ice column extrusion which is driven by the pumping force. Inside the filter, the ice crystals re-crystallise, float and agglomerate on the surface of the mother liquor. This surface is at the beginning of the filter element where an ice-concentrated solution interface is created leading to a growing column of ice crystals as a result of deposition of ice crystals at the interface. The mass flux is directly proportional to the mass flow-rates of the ice slurry for a given ice fraction in the ice slurry. Therefore, throughputrates are proportional to flow-rates. Higher throughputs result in lower residence times which affect the quality of recovered process water for a given process water concentration. (Adeniyi et al., 2013b)

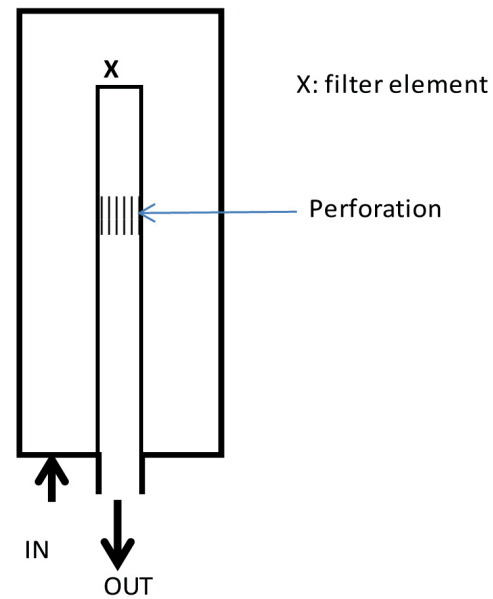

Figure 2

Filter elements of the gravitation HybridICE filter

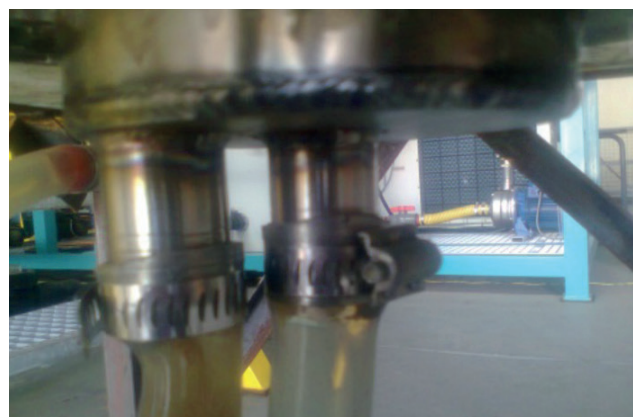

Figure 3

Feed water inlet and outlet for the gravitation HybridICE filter 


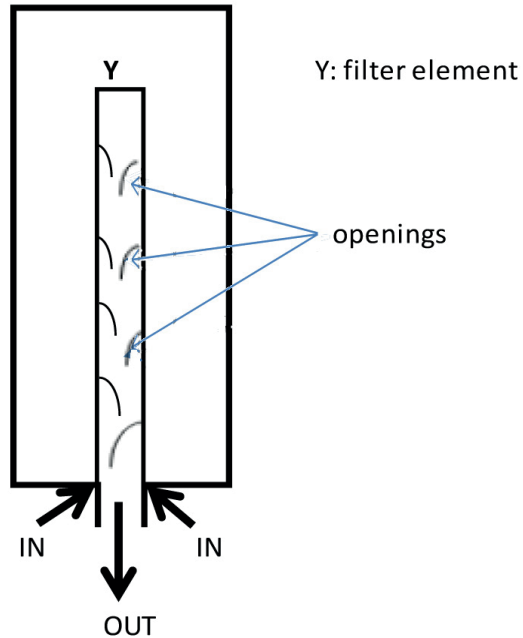

Figure 4

Filter element of the hydraulic HybridICE filter

\section{Hydraulic HybridICE filter}

The Hydraulic HybridICE filter is cylindrical in shape, similar to the gravitation HybridICE filter. The filter elements are also cylindrical. The only difference is the mode of cutting the opening for the concentrated process water to leave the filter. The filtering element has open cuts as shown in Fig. 4, to allow concentrated process water to flow out of the filter.

\section{Mode of feeding slurry into the filter}

As the name of the filter implies, the separation driving force is created as a result of pressure generated by the flow of the slurry. The mode of feeding into the filter has to be able to generate enough pressure to drive the ice column in the filter. The slurry must also be uniformly distributed in the filter. The holes feeding the slurry are smaller in diameter than the major pipe conveying the slurry from the heat exchangers. This is to create extra pressure drive due to sudden reduction of flow. Figure 5 shows the mode of feeding the slurry.

\section{Separation mechanism}

As the slurry flows into the filter, the concentrated process water starts to flow out through the first opening. The formation of the ice column starts from the bottom of the filter and grows upwards until it reaches the height where the ice is harvested using the scraper. The growth of the ice column depends mainly on the ice fraction in the slurry, the surface areas of the existing ice crystals, and Ostwald ripening as a result of residence time in the filter. Overflow is common, chiefly due to formation of the ice column around the opening and also due to the flow into the filter. Although the overflow assists in driving the ice column through the filter, it has to be controlled because of its negative effect on the ice yield, and salt removal in the filter.

\section{Procedure}

The prepared solution was processed in the HybridICE pilot plant. The evaporating temperature of the refrigerant was set

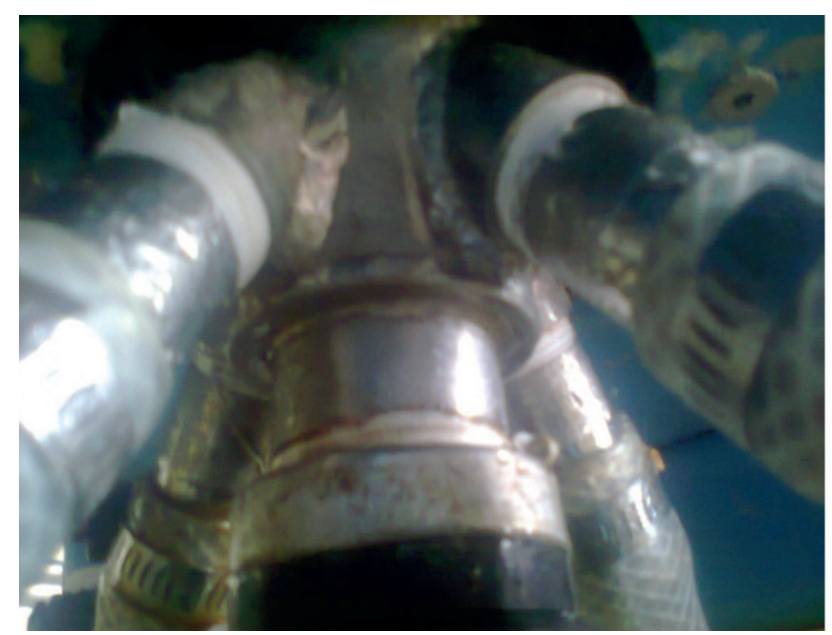

Figure 5

Feed water inlet and outlet for the hydraulic HybridICE filter

at $-10^{\circ} \mathrm{C}$ for each of the heat exchangers because the ambient temperature was high $\left(29^{\circ} \mathrm{C}\right)$ at the time of running the experiment.

\section{Ice fraction}

Samples of slurry were taken from the filter bypass and weighed. The slurry was then filtered using a domestic coffee plunger. The ice part of the slurry was weighed and the ice fraction calculated, as in Eq. (6).

\section{Samples from the hydraulic HybridICE filter}

The slurry was fed into the hydraulic HybridICE filter at a flow rate of $30 \mathrm{l} / \mathrm{min}$. Ice was collected at the filter chute after harvesting with the scraper. Ice was collected with a bucket for 1 minute and the contents weighed to determine the mass yield of ice per minute. This was repeated every 10 minutes from when the ice harvesting started. Samples of ice produced were allowed to melt and attain room temperature before measuring the electrical conductivity.

\section{Samples from the gravitation HybridICE filter}

The slurry was fed into the gravitation HybridICE filter at $10 \mathrm{l} / \mathrm{min}$. Ice was collected manually at the top of the filter by using a string to cut the ice column. Ice was also collected and weighed to determine the mass yield of ice per minute. Samples of ice produced were taken every 10 minutes, and allowed to melt and attain room temperature before measuring the electrical conductivity.

\section{Analytical methods}

The performance of each of the filters was evaluated based on salt removal and ice yield when synthetic wastewater was processed in the pilot plant.

Salt removal was calculated based on the electrical conductivity of the feed solution $C_{\mathrm{f}}$ and the electrical conductivity of the melted ice sample $C_{\mathrm{i}}$. (Adeniyi et al., 2013b)

$$
\text { Salt removal }(\%)=\frac{\left(C_{f}-C_{i}\right)}{C_{f}} \times 100 \%
$$


The yield of ice was based on the mass of ice produced, in kg/ min compared with the ice fraction in the slurry.

$$
\begin{aligned}
& \text { Ice fraction }=\frac{\text { Mass of ice }}{\text { Mass of slurry }} \\
& \text { Yield }(\%)=\frac{\text { Mass of ice per min }}{\text { Ice fraction } \times \text { Mass flow rate of slurry }} \times 100 \%
\end{aligned}
$$

Electrical conductivity measurement was used for the chemical analysis (Wolkersdorfer, 2012). As the electrical conductivity of water is a good indicator of total salinity, it estimates the total amount of solids dissolved in water. A filtration method was used for ice fraction determination (Kousksou et al., 2009).

\section{RESULTS AND DISCUSSION}

\section{Salt removal}

Two major factors affect salt removal in the filter. The first is the quality of the ice in the slurry, and the second is the ice separation mechanism in the filter. One major parameter that affects the quality of the ice in the slurry is the degree of undercooling, which is driven by the difference between the ice point and the evaporating temperature of the refrigerant. For a solution of ice point of $-2^{\circ} \mathrm{C}$, the refrigerant evaporating temperature should be between $-6^{\circ} \mathrm{C}$ and $-8^{\circ} \mathrm{C}$ in order to keep the concentrated solution trapped within the ice bed at the lowest possible level. However, because of the high ambient temperature at the time of the run of this experiment, the evaporating temperature was set at $-10^{\circ} \mathrm{C}$. At this temperature, the tendency for concentrated wastewater inclusion in the ice was high, but ice formation was also favourable. Refrigerant evaporating temperature affects the slurry quality and hence the yield and salt removal in the filter (Adeniyi et al., 2013a). Preliminary testing of the gravitation filter gave a mean of $90 \%$ salt removal from a solution of electrical conductivity of $63.5 \mathrm{mS} / \mathrm{cm}$. At the time when the preliminary test was carried out, the ambient temperature was $14^{\circ} \mathrm{C}$ and the evaporating temperature for the refrigerant was set at $-6^{\circ} \mathrm{C}$ for each of the three heat exchangers. The salt removal will definitely be higher in both filters if the process parameters are controlled. Ulrich and Strege (2002) stated that there is a need to control the crystallisation process in order to ensure product quality of right crystal size, distribution, shape and purity. However, since the purpose was to compare the performance of the two types of filter, we ran at the condition which was conducive for the system at high ambient temperature.

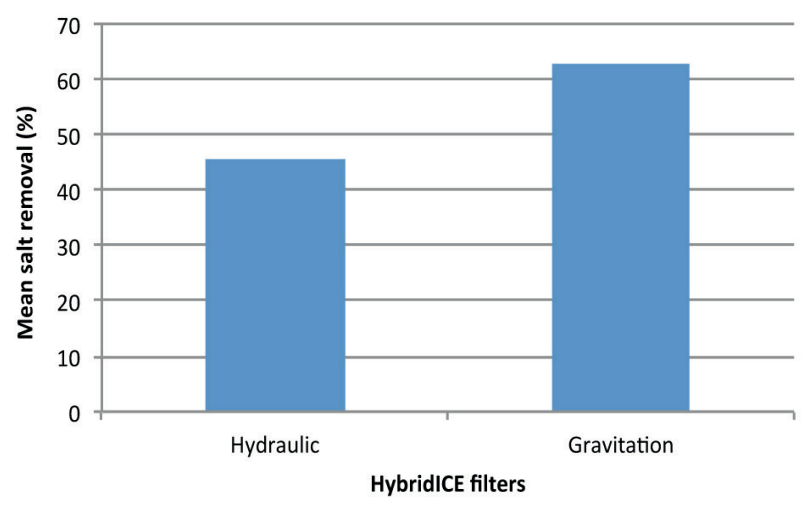

Figure 6

Mean salt removal (no error bars shown)
In the two types of HybridICE filters, there was formation of an ice bed column. Salt removal in the ice bed was similar to that which takes place in sea ice.

Transport mechanisms of brine in sea ice are discussed in the work of Cox and Weeks (1975) and Olsson (1999). Once the ice has been formed, the brine is not static. The transport mechanism of the brine affects the salinity profile during the life cycle of the sea ice. The first mechanism is a thermodynamically driven process where brine pockets migrate from the colder to the warmer regions of the ice. But this mechanism is too slow to cause the changes observed in the salinity profile due to the very slow rate of diffusion in the salt. Brine expulsion disrupts the ice along the basal plane as a result of pressure build up. The pressure gradient drives the brine down and ultimately out of the ice sheet. This is probably the most important desalination mechanism during the initial stages of ice growth. Gravity drainage includes all processes whereby brine, under the influence of gravity, drains out of the ice sheet into the underlying sea water. As an ice sheet increases in thickness, its surface rises higher and higher above sea level to maintain isostatic equilibrium. The accumulation of brine above sea water level thereby produces a pressure head which drives the underlying brine out of the ice. This process, together with flushing, presumably accounts for the low salinity of the upper portion of multilayer sea ice. Flushing is a type of gravity drainage due to the hydrostatic head produced by surface melt water.

The above mechanisms are applicable to the behaviour of the ice bed in the filter, particularly flushing and gravity drainage. However, the time available with sea ice for these mechanisms to significantly affect purity in the ice, is not available in the filter. An increase in residence time, which is the time taken for the ice bed to pass through the filter, can increase salt removal due to recrystallisation of ice in the filter. That is why in the actual sense, the taller the filter, the purer the ice. But this has to be balanced with the ice yield for the process to be economical.

Experimental results showed that salt removal was higher in the gravitation filter than in the hydraulic filter. This was due to the fact that the ice bed in the hydraulic filter started from the bottom of the filter while the concentrated process water flowed out through the perforations on the filter element. Differences in flow caused the ice bed to sit in the concentrated process water and thus affected the overall salt removal. The ice itself was pure but the purity was affected by the concentrated process water around or trapped in the ice bed. There was no interaction between the ice and the concentrated process water in the gravitation filter, except in the stagnant section, so the draining effect on the ice bed was more pronounced.

\section{Ice fraction}

Table 1 shows that the mean ice fraction was $14.9 \%$ and this was used to calculate the mean yield for each of the filters, based on Eq. (6). Although higher ice fractions are desirable, slurry behaviour changes with ice fraction: at up to $25 \%$, the ice slurry flows like conventional chilled water, at 40 to $50 \%$, it exhibits thick slurry characteristics, and at 65 to $75 \%$, the ice slurry has the consistency of soft ice cream (Environmental Process Systems Limited, 2012). Apart from this, high ice fraction is driven by a high temperature gradient which increases the tendency of concentrated solution inclusion in the ice. Based on these facts, it is important to keep the ice fraction below $25 \%$ for good separation in the filters. 
TABLE 1

Ice fraction estimation

\begin{tabular}{|l|c|c|c|}
\hline Sample (no.) & Mass of slurry (g) & Mass of ice (g) & Ice fraction (\%) \\
\hline 1 & 259.3 & 46.7 & 18.0 \\
\hline 2 & 229.1 & 29.1 & 12.7 \\
\hline 3 & 257.8 & 42.5 & 16.5 \\
\hline 4 & 222.1 & 24.9 & 14.7 \\
\hline 5 & 235.4 & 34.6 & 15.3 \\
\hline 6 & 228.8 & 35.0 & 20.2 \\
\hline 7 & 229.0 & 46.3 & 10.6 \\
\hline 8 & 236.5 & 25.1 & 15.6 \\
\hline 9 & 232.3 & 35.3 & 14.8 \\
\hline 10 & 227.8 & 33.7 & $\mathbf{1 4 . 9}$ \\
\hline
\end{tabular}

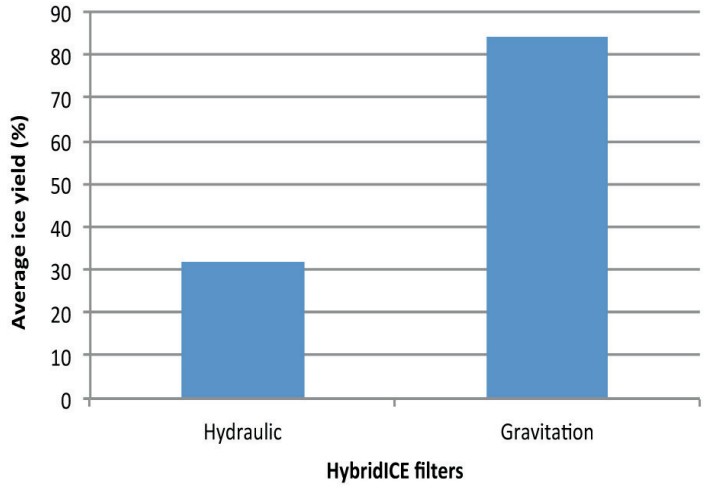

Figure 7

Ice yield

\section{Ice yield}

Ice yield was higher with the gravitation filter than the hydraulic filter. This was because of dead volume (accumulation) in the filter. Ice column formation started at the bottom of the filter and had to grow all the way through the height of the filter before it could be harvested. This was supposed to contribute to the purity of the ice column but for the contamination of the ice column by the concentrated process water due to overflow.

Although the performance of the gravitation HybridICE filter was better than that of the hydraulic HybridICE filter in terms of salt removal and ice yield from the process, it would be necessary to develop both for ice separation in freeze desalination processes. This is because the performance of the hydraulic HybridICE filter can be improved if the flow in the filter is well controlled and the height of the present filter is adjusted. It may be necessary to combine the mechanisms from the two types of filter to form a new type with improved performance. One way to do this is by applying the same type of open cuts in the filter element as in the hydraulic filter, but the cuts must start in an elevated position, as in the gravitation filter.

\section{CONCLUSIONS}

From the results of the experimental work the following conclusions were reached:

- The gravitation HybridICE filter performs better than the hydraulic HybridICE filter in terms of salt removal and ice yield from the process water

- The performance of the hydraulic HybridICE filter is affected by the overflow in the filter and the height of the filter

- Further development can be done to improve the performance of both filters

- The mechanism of ice separation in the two filters can be combined to create a new type of filter with improved performance

\section{ACKNOWLEDGEMENTS}

The authors acknowledge Tshwane University of Technology for providing the research facilities and laboratory equipment, and the THRIP program of the National Research Foundation for funding the project. Mr Frederick S. Oosthuizen is acknowledged, as the holder of the patent on the HybridICE technology. Mr Hennie Joubert is acknowledged for constructing the gravitation HybridICE filter used for the experiment.

\section{REFERENCES}

ADENIYI A, MAREE JP, MBAYA RKK and POPOOLA API (2013b) HybridICE $^{\text {wa }}$ filter design in freeze desalination of mine water. In: Brown A, Figueroa L and Wolkersdorfer C (eds) Reliable Mine Water Technology. Publication Printers, Denver.

ADENIYI A, MAREE JP, MBAYA RKK, POPOOLA API, OOSTHUIZEN FS, MTOMBENI T and ZVINOWANDA CM (2013a) HybridICE ${ }^{\oplus}$ HIF filter: principle and operation, Water Resources Management VII. WIT Trans. Ecol. Environ. 171 1743-3541.

BAKER I, CULLEN D and ILIESCU D (2003) The microstructural location of impurities in ice. Can. J. Phys. 81 1-9

CERCI Y, CENGEL Y, WOOD B, KAHRAMAN N and KARAKAS EN (2003) Improving the thermodynamic and economic efficiencies of desalination plants: minimum work required for desalination and case studies of four working plants. Agreement No. 99-FC-81-0183. Desalination and Water Purification Research and Development Program, Final Report No. 78, November 2003.

CLAYTON R (2011) A review of current knowledge. Desalination for water supply. FR/0013. Foundation for Water Research, Marlow, UK.

COX GFN and WEEKS WF (1975) Brine drainage and initial salt entrapment in sodium chloride ice. U.S. Research Report 345. Army Cold Regions Research and Engineering Laboratory, Hanover, New Hampshire. 
ENVIRONMENTAL PROCESS SYSTEMS LIMITED (2012) Slurry ice application guide. URL: www.epsltd.co.uk/files/slurryice_manual. pdf (Accessed 7 January 2013).

KOUSKSOU T, JAMIL A, ZERAOULI Y and DUMAS JP (2009)

Thermodynamic nonequilibrium in ice-slurry systems. J.

Thermophys. Heat Transfer 232 392-398.

LORAIN O, THIEBAUD P, BADORC E and AURELLE Y (2000) Potential of freezing in wastewater treatment: soluble pollutant applications. Water Res. 35 441-447.

LU Z and XU L (2010) Freezing desalination process. Thermal Desalination Processes - Vol. II. Encyclopedia of Desalination and Water Resources (DESWARE) 2010. URL: http://www.desware.net/ Sample-Chapters/D04/D08-063.pdf (Accessed 6 August 2013).

MAURER M, PRONK W and LARSEN TA (2006) Treatment processes for source-separated urine. Water Res. 40 3151-3166.

MTOMBENI T, MAREE JP, ZVINOWANDA CM, ASANTE JKO, OOSTHUIZEN FS and LOUW WJ (2013) Evaluation of the performance of a new freeze desalination technology. Int. J. Environ. Sci. Technol. 10 (3) 545-550.
OLSSON K (1999) Uptake, re-distribution and release of contaminants by sea ice. Akvaplan-niva Report No: APN-414.1672.001.99. Norwegian Polar Institute, Tromsø.

QIN FGF, CHEN XD, PREMATHILAKA S and FREE K (2008) Experimental study of wash columns used for separating ice from ice-slurry. Desalination 219 223-228.

RAHMAN MS, AHMED M and DON CX (2006) Freezing-melting process and desalination: i. Review of the state-of-the-art. Sep. Purif. Rev. 35 59-96.

SHONE RDC (1987) The freeze desalination of mine waters. J. S. Afr. Inst. Min. Metall. 87 107-112.

ULRICH J and STREGE C (2002) Some aspects of the importance of metastable zone width and nucleation in industrial crystallizers. J. Cryst. Growth 237-239 2130-2135.

WOLKERSDORFER C (2012) Getting rid of chemical analysis - Mine water management with indicator parameters. In: McCullough CD, Lund MA and Wyse L (eds) International Mine Water Association Symposium, Bunbury (Edith Cowan University). 505-512. 